\title{
Endosonographic evaluation of c-kit-positive gastrointestinal stromal tumor
}

T. Okai, T. Minamoto, K. Ohtsubo, M. Mai, and N. Sawabu

Background: Endoscopic ultrasonography (EUS) is a valuable imaging tool of the gastrointestinal mesenchymal tumor because it allows demonstrating a hypoechoic mass that is contiguous with the forth hypoechoic layer of the normal gut wall. However, the studies are still a few, and the ability of EUS to distinguish between benign and malignant mesenchymal tumors remained to be confirmed. In the present study we designed to evaluate the endosonographic features of benign and malignant c-kit-positive gastrointestinal stromal tumors (GISTs) in comparison with those of leiomyomas and schwannomas.

Methods: Twenty-four patients with gastric mesenchymal tumors who underwent EUS and surgical treatment were enrolled. GISTs were defined as c-kit (CD117)-positive tumors, leiomyomas as desmin-positive and c-kit-negative tumors, and schwannomas as S-100positive and c-kit-negative tumors. Invasion to adjacent organs or more than 20 mitotic counts per 50 high power fields indicated malignancy.

Results: There were 19 GISTs, three leiomyomas, and two schwannomas. All five malignant tumors were GISTs. A marginal halo was found in 12 of 19 GISTs and in both of the schwannomas, but not in any of the three leiomyomas. The echogenicities of GISTs were low but higher than that of the normal proper muscle layer, whereas those of leiomyomas and schwannomas were usually low. Lobulation of the tumor surface was documented only in GISTs, particularly in malignant ones (figure). The tumor doubling time of a malignant GIST was 9.3 months, and that of six benign GISTs was 18.7 months (range $=10.7-28.0$ months).

Conclusions: Marginal halo and relatively higher echogenicity on EUS might suggest GIST. Marginal lobulation and a short doubling time may be signs of a malignant GIST.

Figure:

Comparison of EUS features between benign/borderline and malignant GISTs.

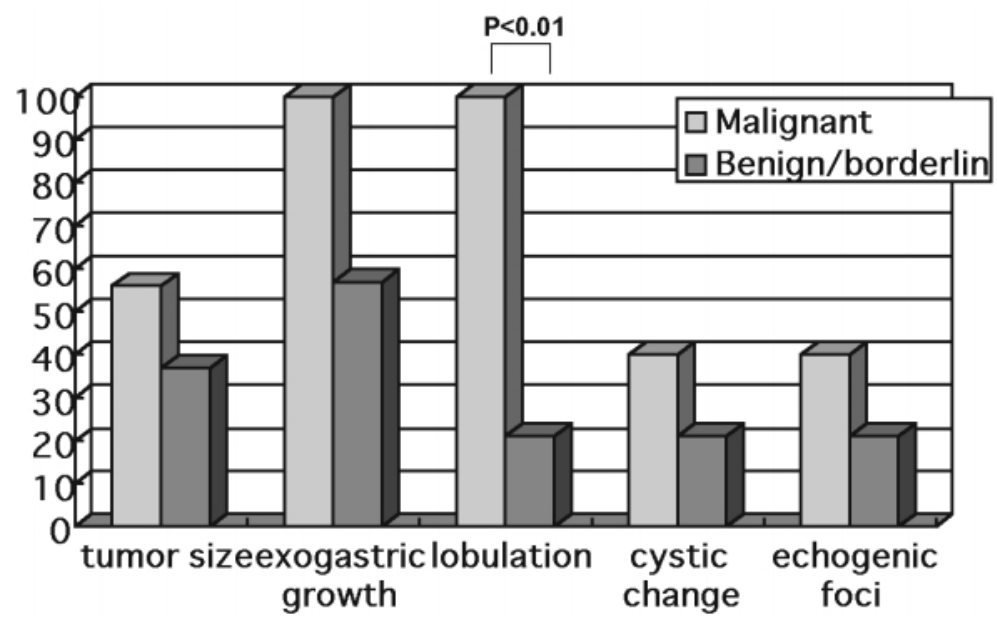

